PROCEEDINGS OF THE

AMERICAN MATHEMATICAL SOCIETY

Volume 129, Number 6, Pages 1563-1572

S 0002-9939(01)05565-4

Article electronically published on February 2, 2001

\title{
FREE SUMMANDS OF CONORMAL MODULES AND CENTRAL ELEMENTS IN HOMOTOPY LIE ALGEBRAS OF LOCAL RINGS
}

\author{
SRIKANTH IYENGAR
}

(Communicated by Wolmer V. Vasconcelos)

\begin{abstract}
If $(Q, \mathfrak{n}) \rightarrow(R, \mathfrak{m})$ is a surjective local homomorphism with kernel $I$, such that $I \subseteq \mathfrak{n}^{2}$ and the conormal module $I / I^{2}$ has a free summand of rank $n$, then the degree 2 central subspace of the homotopy Lie algebra of $R$ has dimension greater than or equal to $n$. This is a corollary of the Main Theorem of this note. The techniques involved provide new proofs of some well known results concerning the conormal module.
\end{abstract}

Let $R$ be a noetherian local ring with maximal ideal $\mathfrak{m}$ and residue field $k$. Ring theoretic properties of $R$ are reflected on the algebra structures carried by $\operatorname{Tor}^{R}(k, k)$ and $\operatorname{Ext}_{R}(k, k)$. Recall that the former has the $\pitchfork$-product of Cartan and Eilenberg, and the latter the Yoneda multiplication. The $k$-algebra structure of $\operatorname{Tor}^{R}(k, k)$ is rather "simple": It is free in the appropriate category, and so determined by the dimension of the $k$-vector space $\operatorname{Tor}_{i}^{R}(k, k)$, for all integers $i \geq 0$, that is to say, the Betti numbers of $k$ over $R$. The multiplicative structure of $\operatorname{Ext}_{R}(k, k)$ is quite another matter.

The simplicity of the product on $\operatorname{Tor}^{R}(k, k)$ arises from the fact that it is endowed with additional structures: It is a commutative algebra, in the graded sense, with a family of divided powers, and has a diagonal map that is compatible with the divided powers algebra structure on it. In other words, the $k$-algebra $\operatorname{Tor}^{R}(k, k)$ is a commutative Hopf algebra with divided powers, and so is free as a divided powers algebra.

In contrast, the multiplication on $\operatorname{Ext}_{R}(k, k)$ is decidedly non-commutative, unless $R$ happens to be a complete intersection of a special kind. The graded $k$ dual of the product on $\operatorname{Tor}^{R}(k, k)$ turns $\operatorname{Ext}_{R}(k, k)=\operatorname{Tor}^{R}(k, k)^{*}$ into a Hopf algebra. Attention has focussed on a certain subspace of primitives of this Hopf algebra, denoted $\pi(R)$, which is a Lie algebra with the bracket operation defined by the commutator in $\operatorname{Ext}_{R}(k, k)$. This object is called the homotopy Lie algebra of $R$. The importance of this Lie algebra is attested to by its defining property: Its universal enveloping algebra is $\operatorname{Ext}_{R}(k, k)$.

In this note, we are concerned with the centre of the homotopy Lie algebra of $R$, denoted $\zeta(R)$. This subspace, besides being a measure of the non-commutativity of $\pi(R)$, and hence of $\operatorname{Ext}_{R}(k, k)$, plays an important part in the change of rings

Received by the editors April 7, 1999 and, in revised form, May 12, 1999.

1991 Mathematics Subject Classification. Primary 13C15, 13D03, 13D07, 18G15.

Key words and phrases. Homotopy Lie algebra, central elements, conormal module. 
properties of the homotopy Lie algebra, among other things; the interested reader is referred to [3], and the references therein, for additional information on this matter. The construction of $\pi(R)$ outlined above is a compilation of work of André, Assmus, Avramov, Gulliksen, Levin, Sjödin, Schoeller, and others.

Our primary goal is to establish the following

Main Theorem. Let $(Q, \mathfrak{n}, k)$ be a local ring and $I$ an ideal in $Q$. Set $R=Q / I$. If there is an ideal $J$ in $Q$ with $I^{2} \subseteq J \subseteq I$ and the $R$-module $I / J$ is free, then

$$
\operatorname{rank}_{k} \zeta^{2}(R) \geq \operatorname{rank}_{R}\left(\frac{I}{J}\right)-\operatorname{rank}_{k}\left(\frac{I}{J+I \cap \mathfrak{n}^{2}}\right) .
$$

Here is a special case of the theorem: If the ideal $I$ is generated by a regular sequence, then $I / I^{2}$ is a free $R$-module, so that

$$
\operatorname{rank}_{k} \zeta^{2}(R) \geq \operatorname{rank}_{R}\left(\frac{I}{I^{2}}\right)-\operatorname{rank}_{k}\left(\frac{I}{I \cap \mathfrak{n}^{2}}\right) .
$$

This well known fact has the following consequence: If the m-adic completion $\widehat{R}$ of a local ring $(R, \mathfrak{m}, k)$ is such that $\widehat{R} \cong Q /(f)$ with $(Q, \mathfrak{n}, k)$ a local ring and $f \in \mathfrak{n}^{2}$ a non-zero divisor, in other words, if $\widehat{R}$ has an embedded deformation, then $\pi^{2}(\widehat{R})$ has a central element, and since $\pi(R) \cong \pi(\widehat{R})$, there is a central element in $\pi^{2}(R)$. In 4], Avramov posed the following question: Does every element in $\zeta^{\geqslant 2}(R)$ arise from an embedded deformation of $\widehat{R}$ ?

The graded version of this question has been settled in the negative: In [5], Dupont exhibits a graded ring $R$ with a central element in $\pi^{2}(R)$ and proves that there is no graded ring $Q$ with a homogeneous non-zero divisor $f$ such that $R=$ $Q /(f)$. The local case of this problem remains open, although Löfwall [10] and Avramov [4] have answered it in the affirmative in certain special situations.

One of the difficulties in addressing the question above is that, hitherto, other than embedded deformations, one had no good source of central elements in $\pi(R)$. The theorem above is offered as procedure for generating rings with central elements in the homotopy Lie algebra which do not a priori arise from embedded deformations. It should be noted that this result builds on the work in [5].

The proof of the Main Theorem is given in Section 1. As an outgrowth of these investigations, we found another approach to certain well known results regarding the conormal module $I / I^{2}$. These are discussed in Section 2.

\section{Central elements}

The proofs in this paper rely on computations with Differential Graded algebras with divided powers, henceforth abbreviated to DG $\Gamma$-algebras. We refer to the monographs of Gulliksen and Levin [7], and Avramov [3], for definitions and basic results concerning these objects. We adhere to the following conventions:

1.1. Let $Q$ be a commutative ring. A $D G \Gamma$-algebra over $Q$ is a DG $\Gamma$-algebra $A$ such that $A_{0}$ is a $Q$-algebra. Any DG $\Gamma$-algebra $A$ considered here will be such that the ring $A_{0}$ is noetherian and each $A_{i}$ is a finitely generated $A_{0}$-module; in particular, all rings, which we regard as DG $\Gamma$-algebras concentrated in degree 0 , will be noetherian.

For a DG $\Gamma$-algebra $A$, the symbol $A\langle X\rangle$ denotes a DG $\Gamma$-algebra obtained by successive adjunction of exterior and divided power variables; cf. [3] 6]. In this case, 
for any integer $n$, we write $X_{n}$ for the variables of degree $n$, and $A\left\langle X_{\leqslant n}\right\rangle$ to denote the DG $\Gamma$-subalgebra of $A\langle X\rangle$ obtained by adjunction of variables of degree $\leq n$.

A derivation of a $\Gamma$-algebra $A$ is a homogeneous $Q$-linear map $\theta: A \rightarrow A$ such that

$$
\begin{aligned}
& \theta(x y)=\theta(x) y+(-1)^{|\theta||x|} x \theta(y) \quad \text { for } \quad x, y \in A ; \\
& \theta\left(x^{(i)}\right)=\theta(x) x^{(i-1)} \quad \text { for } \quad x \in A_{\text {even }} \text { and all } i \geq 1 .
\end{aligned}
$$

When we refer to a derivation of a DG $\Gamma$-algebra, it is to be understood that it is a derivation of the underlying $\Gamma$-algebra, and, unless stated to the contrary, that it commutes with the differential: $\partial \circ \theta=(-1)^{|\theta|} \theta \circ \partial$.

For ease of reference, we record the following fact about derivations; cf. [7] 1.3.2].

1.2. A derivation $\theta$ on a DG $\Gamma$-algebra $A$ extends to $A\langle X| \partial x \in A$, for $x \in X\rangle$ if and only if $\theta(\partial x) \in \partial(A)$ for each $x \in X$. If $\theta(\partial x)=\partial\left(b_{x}\right)$, then one may extend $\theta$ to $A\langle X\rangle$ with $\theta(x)=(-1)^{|\theta|} b_{x}$.

Next, we list various criteria that allow one to detect the existence of direct summands in the conormal module $I / I^{2}$; verifying these assertions is a routine exercise. Recall that the free rank of an $R$-module $M$ is the maximal rank of a free direct summand of $M$; we denote it f-rank ${ }_{R} M$.

1.3. Consider an ideal $I$ in a local $\operatorname{ring} Q$. Set $R=Q / I$.

(1) f-rank $_{R}\left(I / I^{2}\right) \geq n$ if and only if there is an ideal $J$ in $Q$ with $I^{2} \subseteq J \subseteq I$ such that the $R$-module $I / J$ is free of rank $n$;

(2) the classes of $a_{1}, \ldots, a_{n} \in I$ form a basis for a free summand of $I / I^{2}$ if and only if they extend to a minimal set of generators $a_{1}, \ldots, a_{n}, b_{1}, \ldots, b_{m}$ for $I$ with the property that for any $r_{1}, \ldots, r_{n}$ in $Q$

$$
\sum_{i=1}^{n} r_{i} a_{i} \in\left(b_{1}, \ldots, b_{m}\right) \Longrightarrow r_{j} \in I \quad \text { for } \quad 1 \leq j \leq n .
$$

The following proposition adds to this list; it is a particular case of [3, 6.2.7]. We give a direct proof suited to the situation on hand. First, a piece of notation: For integers $i, j$, we set

$$
\delta_{i j}= \begin{cases}1 & \text { if } i=j \\ 0 & \text { otherwise }\end{cases}
$$

1.4. Proposition. Let $Q$ be a ring, $I \subset Q$ a proper ideal, and $Q\langle X\rangle$ a $D G \Gamma$ -

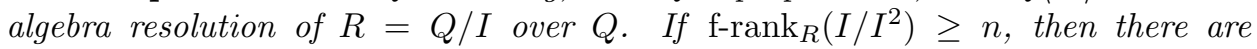
elements $x_{1}, \ldots, x_{n} \in Q\langle X\rangle$ of degree 1 , and $Q$-linear derivations $\theta_{i}$ of $Q\langle X\rangle$ such that $\theta_{i}\left(x_{j}\right)=\delta_{i j}$ for $1 \leq i, j \leq n$.

If the classes of $a_{1}, \ldots, a_{n}$ form a basis for a free direct summand of $I / I^{2}$, then the $x_{i}$ may be chosen with $\partial\left(x_{i}\right)=a_{i}$ for $1 \leq i \leq n$.

Proof. Let $a_{1}, \ldots, a_{n}, b_{1}, \ldots, b_{m}$ be the minimal generating set for the ideal $I$ provided by 1.3]2. Since $\partial\left(X_{1}\right)$ generates the ideal $I$, by a linear change of variables we may assume that $X_{1}=\left\{x_{1}, \ldots, x_{n}, y_{1}, \ldots, y_{m+p}\right\}$ with $\partial\left(x_{i}\right)=a_{i}$, for $1 \leq i \leq n$, and $\partial\left(y_{j}\right)=b_{j}$, for $1 \leq j \leq m$, and $\partial\left(y_{m+k}\right)=0$, for $1 \leq k \leq p$.

For $1 \leq i \leq n$, set $\theta_{i}: Q \rightarrow Q$ to be the trivial $Q$-linear derivation with $\theta_{i}(r)=0$ for all $r \in Q$. By 1.2 these extend to $Q$-linear $\Gamma$-derivations on $Q\left\langle X_{1}\right\rangle$ with $\theta_{i}\left(x_{j}\right)=$ $\delta_{i j}$ and $\theta_{i}\left(y_{k}\right)=0$. We prove that these extend to $Q\langle X\rangle$. 
To begin with, for any element $x \in X_{2}$, one has $\partial x=\left(\sum_{i=1}^{n} r_{i} x_{i}\right)+y$, for some elements $r_{1}, \ldots, r_{n}$ in $Q$ and $y \in \sum_{j=1}^{m+p} Q y_{j}$. Since $\partial^{2}(x)=0$, one has

$$
\sum_{i=1}^{n} r_{i} a_{i} \in \sum_{j=1}^{m+p} Q \partial\left(y_{j}\right)=\left(b_{1}, \ldots, b_{m}\right) .
$$

From [1.3. 2 we derive that the elements $r_{1}, \ldots, r_{n}$ are in $I$, and so $\theta_{i}(\partial(x))=$ $r_{i} \in \partial\left(Q\left\langle X_{1}\right\rangle\right)$. Thus, by 1.2 the $\theta_{i}$ extend to $\Gamma$-derivations on $Q\left\langle X_{\leqslant 2}\right\rangle$.

Now assume that the $\theta_{i}$ have been extended to $Q\left\langle X_{\leqslant k}\right\rangle$, for some $k \geq 2$. For any $x \in X_{k+1}$ the element $\theta_{i}(\partial x)$ in $Q\left\langle X_{\leqslant k}\right\rangle$ is a cycle:

$$
\partial \theta_{i}(\partial x)=-\theta_{i} \partial(\partial x)=0 \text {. }
$$

Since the degree of $\theta_{i}(\partial x)$ is $k-1 \geq 1$, and $Q\left\langle X_{\leqslant k}\right\rangle$ has no homology in degrees $1, \ldots, k-1$, we find that $\theta_{i}(\partial x) \in \partial\left(Q\left\langle X_{\leqslant k}\right\rangle\right)$. Thus, by 1.2 , the $\theta_{i}$ extend to derivations on $Q\left\langle X_{\leqslant k+1}\right\rangle$. A direct limit argument completes the proof.

The proof of the Main Theorem hinges on a well known link between the Lie algebra $\pi(R)$ and the action of certain derivations of the acyclic closure of $R$; cf. [3, 6.3.1], or [7, 1.9], for the details on acyclic closures. We give a precis of this relationship in a form suited to our use, modeled along the lines of [3, 10.2.1].

1.5. Let $A$ be a DG $\Gamma$-algebra such that $A_{0}$ is a local ring, with maximal ideal $\mathfrak{m}$ and residue field $k$. As in the case of local rings, the $k$-algebra $\operatorname{Ext}_{A}(k, k)$ is the universal enveloping algebra of a graded Lie algebra, which is unique up to isomorphism, and it is denoted $\pi(A)$.

Let $A\langle X\rangle$ be an acyclic closure of $k$ over $A$, where $X=\left\{x_{i}\right\}_{i \geqslant 1}$ is ordered such that $\left|x_{i}\right| \leq\left|x_{j}\right|$ for $i<j$. The $A$-module $D$ consisting of all the $A$-linear $\Gamma$ derivations of $A\langle X\rangle$ is a DG Lie algebra - here we do not assume that the derivations commute with the differential on $A\langle X\rangle$-with Lie bracket defined by

$$
[\theta, \vartheta]=\theta \circ \vartheta-(-1)^{|\theta||\vartheta|} \vartheta \circ \theta .
$$

The following statements hold:

(1) $\pi(A) \cong \mathrm{H}(D)$ as Lie algebras;

(2) for $i \geq 1$, there are derivations, $\vartheta_{i} \in D$, that commute with the differential on $A\langle X\rangle$, with $\vartheta_{i}\left(x_{j}\right)=\delta_{i j}$ for $j \leq i$;

(3) each set of derivations $\left\{\vartheta_{i}\right\}_{i \geqslant 1}$ that satisfy condition (2) maps to a $k$-basis for $\mathrm{H}(D)$.

In the light of these results, henceforth we identify $\pi(A)$ with $\mathrm{H}(D)$.

1.6. Following André, we say that a cycle $z$ in a DG $\Gamma$-algebra $B$ is a special cycle if there is a derivation $\theta: B \rightarrow B$ such that $\theta(z)=1$. Our interest in special cycles arises from a result established by André [1] which, tailored to our needs, reads:

If $z_{1}, \ldots, z_{n}$ are special cycles in a DG $\Gamma$-algebra $B$, with associated derivations $\theta_{1}, \ldots, \theta_{n}$, then there is a DG $\Gamma$-subalgebra $A \subseteq B$ such that

(i) $\bigcap_{i=1}^{n} \operatorname{Ker}\left(\theta_{i}\right) \subseteq A$;

(ii) the morphism of DG $\Gamma$-algebra, extending the inclusion $A \subseteq B$

$$
A\left\langle x_{1}, \ldots, x_{n} \mid \partial\left(x_{i}\right)=0\right\rangle \rightarrow B \quad \text { with } \quad x_{i} \mapsto z_{i},
$$

is an isomorphism.

When we encounter such a situation, we write $B=A\left\langle z_{1}, \ldots, z_{n}\right\rangle$, although this constitutes an abuse of notation. 
For our proof of the Main Theorem, it is expedient to isolate the following

1.7. Observation. Consider an ideal $I$ in a local $\operatorname{ring}(Q, \mathfrak{n}, k)$ such that $I^{2}=0$ and the $Q / I$-module $I$ is free. Set $\mathfrak{m}=\mathfrak{n} / I$, and

$$
n=\mu(I)-\operatorname{rank}_{k}\left(\frac{I}{I \cap \mathfrak{n}^{2}}\right) \text {. }
$$

There are elements $a_{1}, \ldots, a_{n}, b_{1}, \ldots, b_{m}$, and $r_{1}, \ldots, r_{e}$ in $\mathfrak{n}$ such that

(i) $a_{1}, \ldots, a_{n}$ minimally generate $I \cap \mathfrak{n}^{2}$;

(ii) $a_{1}, \ldots, a_{n}, b_{1}, \ldots, b_{m}$ is a minimal set of generators for $I$;

(iii) $r_{1}, \ldots, r_{e}$ map to a minimal set of generators for $\mathfrak{m}$;

(iv) $\mathfrak{n}=\left(r_{1}, \ldots, r_{e}, b_{1}, \ldots, b_{m}\right)$.

Indeed, from the exact sequence of $k$-vector spaces

$$
0 \rightarrow \frac{I \cap \mathfrak{n}^{2}}{\mathfrak{n} I} \rightarrow \frac{I}{\mathfrak{n} I} \rightarrow \frac{I}{I \cap \mathfrak{n}^{2}} \rightarrow 0
$$

we deduce that the rank of the $k$-vector space $\left(I \cap \mathfrak{n}^{2}\right) / \mathfrak{n} I$ is $n$. Select elements $a_{1}, \ldots, a_{n}$ in $I \cap \mathfrak{n}^{2}$ which map to a basis for $\left(I \cap \mathfrak{n}^{2}\right) / \mathfrak{n} I$, and elements $b_{1}, \ldots, b_{m}$ in $I$ which map to a basis for $I /\left(I \cap \mathfrak{n}^{2}\right)$. The exact sequence above implies that $a_{1}, \ldots, a_{n}, b_{1} \ldots, b_{m}$ is a minimal system of generators for $I$. Conditions (a) and (b) are then fulfilled. Now, pick elements $r_{1}, \ldots, r_{e}$ which map to a minimal set of of generators for $\mathfrak{m}$. Note that

$$
\begin{aligned}
\mathfrak{n} & =\left(r_{1}, \ldots, r_{e}\right)+I \\
& =\left(r_{1}, \ldots, r_{e}\right)+\left(b_{1}, \ldots, b_{m}\right)+\left(a_{1}, \ldots, a_{n}\right) \\
& =\left(r_{1}, \ldots, r_{e}\right)+\left(b_{1}, \ldots, b_{m}\right)
\end{aligned}
$$

where the second equality is by (b), and the third follows from Nakayama's lemma, since $\left(a_{1}, \ldots, a_{n}\right) \subseteq \mathfrak{n}^{2}$. This justifies (d).

1.8. Proof of the Main Theorem. Consider the ring $\bar{Q}=Q / J$, and the ideal $\bar{I}=I / J$ in $\bar{Q}$. Observe that $R=\bar{Q} / \bar{I}$ with $(\bar{I})^{2}=0$, and that the $R$-module $\bar{I}$ is free. Moreover, with $\overline{\mathfrak{n}}=\mathfrak{n} / J$, one has an isomorphism

$$
\frac{\bar{I}}{\bar{I} \cap(\overline{\mathfrak{n}})^{2}} \cong \frac{I}{J+I \cap \mathfrak{n}^{2}} .
$$

Therefore, by descending to the $\operatorname{ring} \bar{Q}$ we assume that $J=0$, so that $I^{2}=0$ and $I$ is a free $R$-module. This places us in the situation of Observation 1.7 For the rest of this proof we utilize the notation and conclusion of the same.

Let $Q\langle X\rangle \rightarrow R$ be a DG $\Gamma$-resolution of $R$ over $Q$. By definition, the canonical map

$$
\pi(R) \rightarrow \pi(Q\langle X\rangle)
$$

is an isomorphism of Lie algebras. Thus, it suffices to establish that the rank of $\zeta^{2}(Q\langle X\rangle)$ is at least $n$.

The elements $r_{1}, \ldots, r_{e}$ map to a minimal set of generators for the maximal ideal of $R$, so that the first stage in the acyclic closure of $k$ over $Q\langle X\rangle$ is given by

$$
Q\left\langle X, Y_{1}\right\rangle=Q\langle X\rangle \otimes_{Q} Q\left\langle y_{1}, \ldots, y_{e} \mid \partial\left(y_{i}\right)=r_{i}\right\rangle
$$

Since $a_{1}, \ldots, a_{n}, b_{1}, \ldots, b_{m}$ is a minimal system of generators for $I$, there are elements $x_{1}, \ldots, x_{n+m} \in X_{1}$ with $\partial\left(x_{i}\right)=a_{i}$, for $1 \leq i \leq n$, and $\partial\left(x_{n+j}\right)=b_{j}$, 
for $1 \leq j \leq m$. From part (d) of the observation above we obtain $a_{i} \in \mathfrak{n}=$ $\left(r_{1}, \ldots, r_{e}, b_{1}, \ldots, b_{m}\right)$, for $1 \leq i \leq n$, and hence there are elements

$$
c_{i} \in \sum_{j=1}^{e} Q y_{j}+\sum_{k=1}^{m} Q x_{n+k} \quad \text { with } \quad \partial\left(c_{i}\right)=a_{i} .
$$

Therefore, the elements $z_{i}=x_{i}-c_{i}$, for $1 \leq i \leq n$, are cycles in $Q\left\langle X, Y_{1}\right\rangle$.

Let $\theta_{1}, \ldots, \theta_{n}$ be the $Q$-linear derivations of $Q\langle X\rangle$ supplied by Proposition 1.4 corresponding to the elements $a_{1}, \ldots, a_{n}$. Each $\theta_{i}$ extends to a $Q\left\langle Y_{1}\right\rangle$-linear derivation $\widetilde{\theta}_{i}: Q\left\langle X, Y_{1}\right\rangle \rightarrow Q\left\langle X, Y_{1}\right\rangle$. Note that for $1 \leq i, j \leq n$ and $1 \leq k \leq e$ one has

$$
\widetilde{\theta}_{i}\left(z_{j}\right)=\delta_{i j} \text { and } \tilde{\theta}_{i}\left(y_{k}\right)=0
$$

In particular, the elements $z_{1}, \ldots, z_{n}$ are special cycles of degree 1 in $Q\left\langle X, Y_{1}\right\rangle$.

From this point onwards the proof develops along the lines of that of [, 1]. Given the $\widetilde{\theta}_{i}$, one invokes 1.6 to write

$$
Q\left\langle X, Y_{1}\right\rangle=A\langle Z\rangle=A\left\langle z_{1}, \cdots, z_{n} \mid \partial\left(z_{i}\right)=0\right\rangle
$$

where $A$ is a DG $\Gamma$-subalgebra of $Q\left\langle X, Y_{1}\right\rangle$ containing $y_{1}, \ldots, y_{e}$. Note that $\mathrm{H}_{0}(A)=$ $k$. Let $A\left\langle Y_{\geqslant 2}\right\rangle=A\left\langle y_{e+1}, y_{e+2}, \ldots\right\rangle$ be an acyclic closure of $k$ over $A$, with $\left|y_{i}\right| \leq\left|y_{j}\right|$ if $i<j$. Set

$$
Q\langle X, Y\rangle=Q\left\langle X, Y_{1}, Y_{\geqslant 2}\right\rangle=Q\left\langle X, Y_{1}\right\rangle \otimes_{A} A\left\langle Y_{\geqslant 2}\right\rangle
$$

Observe that the DG $\Gamma$-algebra $Q\langle X, Y\rangle=A\left\langle Y_{\geqslant 2}\right\rangle\langle Z\rangle$ is quasi-isomorphic to $k\langle Z\rangle$, so that

$$
Q\langle X, Y, U\rangle=Q\langle X, Y\rangle\left\langle u_{1}, \ldots, u_{n} \mid \partial\left(u_{i}\right)=z_{i}\right\rangle
$$

is an acyclic closure of $k$ over $Q\langle X\rangle$. Now, by [1.5]3, derivations corresponding to the variables in $Y \cup U$, that satisfy condition 1.5, form a basis for $\pi(Q\langle X\rangle)$.

Claim. The variables $u_{1}, \ldots, u_{n}$ correspond to central elements in $\pi^{2}(Q\langle X\rangle)$.

It suffices to construct $Q\langle X\rangle$-linear derivations $\left\{\omega_{i}\right\}_{i=1}^{n}$ and $\left\{\vartheta_{j}\right\}_{j \geqslant 1}$, of the DG $\Gamma$-algebra $Q\langle X, Y, U\rangle$ such that

(i) $\omega_{i}\left(u_{j}\right)=\delta_{i j}$ for $u_{j} \in U$ and $\omega_{i}\left(A\left\langle Y_{\geqslant 2}\right\rangle\right)=0$;

(ii) $\vartheta_{j}\left(y_{j}\right)=1$ and $\vartheta_{j}(v) \in A\left\langle Y_{\geqslant 2}\right\rangle$ for $v \in Y \cup U$.

Indeed, if such $\omega_{i}$ and $\vartheta_{j}$ exist, then, according to 1.5, the classes of these elements form a basis for $\pi(Q\langle X\rangle)$. For any $1 \leq i \leq n$ and $v \in Y \cup U$, one has

$$
\begin{aligned}
{\left[\omega_{i}, \vartheta_{j}\right](v) } & =\omega_{i}\left(\vartheta_{j}(v)\right)-\vartheta_{j}\left(\omega_{i}(v)\right) \\
& =\omega_{i}\left(\vartheta_{j}(v)\right) \\
& =0
\end{aligned}
$$

where the second equality holds since, by $(\mathrm{a}), \omega_{i}(v) \in\{0,1\}$, and the third equality follows from (a) and (b). Since $\left[\omega_{i}, \vartheta_{j}\right]$ is a $Q\langle X\rangle$-linear derivation, this entails $\left[\omega_{i}, \vartheta_{j}\right]=0$ on $Q\langle X, Y, U\rangle$. A similar computation proves that $\left[\omega_{i}, \omega_{j}\right]=0$ for $1 \leq j \leq n$, so that the class of $\omega_{i}$ is central in $\pi(Q\langle X\rangle)$ (n.b. $\left|\omega_{i}\right|=2$ for each $i$ ).

It remains to construct derivations that satisfy the conditions listed above.

For each element $u_{i} \in U$, one has $\partial\left(u_{i}\right) \in Q\langle X, Y\rangle$, so that we define $\omega_{i}$ to be the $Q\langle X, Y\rangle$-linear derivation of $Q\langle X, Y, U\rangle$ with $\omega_{i}\left(u_{j}\right)=\delta_{i j}$. This $\omega_{i}$ has the required properties. 
Fix an integer $j \geq 1$. Let $\vartheta_{j}$ be the $Q\langle X\rangle$-linear derivation on $Q\langle X\rangle\left\langle y_{1}, \ldots, y_{j}\right\rangle$ defined by $\vartheta_{j}\left(y_{k}\right)=\delta_{j k}$, for $k \leq j$. We resort to an induction argument to extend this derivation to $Q\langle X, Y\rangle$ with the property that $\vartheta_{j}\left(A\left\langle Y_{\geqslant 2}\right\rangle\right) \subseteq A\left\langle Y_{\geqslant 2}\right\rangle$.

Since the variables $y_{1}, \ldots, y_{e}$ are contained in $A \subset Q\left\langle X, Y_{1}\right\rangle$, we may write $A=A^{\prime}\left\langle y_{1}, \ldots, y_{e}\right\rangle$ with $A^{\prime}$ a DG $\Gamma$-subalgebra of $Q\langle X\rangle$. In particular, $\vartheta_{j}\left(A^{\prime}\right)=0$, so that $\vartheta_{j}\left(A^{\prime}\left\langle y_{1}, \ldots, y_{j}\right\rangle\right) \subseteq A^{\prime}\left\langle y_{1}, \ldots, y_{j}\right\rangle$. This is the basis of our induction. Now assume that $\vartheta_{j}$ has been defined on $y_{i}$ for all $i \leq k$ in such a manner that $\vartheta_{j}\left(A^{\prime}\left\langle y_{1}, \ldots, y_{k}\right\rangle\right) \subseteq A^{\prime}\left\langle y_{1}, \ldots, y_{k}\right\rangle$. By construction, $\partial\left(y_{k+1}\right) \in A^{\prime}\left\langle y_{1}, \ldots, y_{k}\right\rangle$, and the induction hypothesis yields that the element $\vartheta_{j}\left(\partial\left(y_{k+1}\right)\right)$ is a cycle in $A^{\prime}\left\langle y_{1}, \ldots, y_{k}\right\rangle$. Since $A^{\prime}\langle Y\rangle=A\left\langle Y_{\geqslant 2}\right\rangle$ is acyclic, there is an element $v \in A^{\prime}\langle Y\rangle$ such that $\partial(v)=\vartheta_{j}\left(\partial\left(y_{k+1}\right)\right)$; the degree of $\partial(v)$ entails $v \in A^{\prime}\left\langle y_{1}, \ldots, y_{k}\right\rangle$. Thus, by 1.2. one can extend $\vartheta_{j}$ with $\vartheta_{j}\left(y_{k+1}\right)=(-1)^{\left|\vartheta_{j}\right|} v$, and so $\vartheta_{j}\left(A^{\prime}\left\langle y_{1}, \ldots, y_{k+1}\right\rangle\right) \subseteq$ $A^{\prime}\left\langle y_{1}, \ldots, y_{k+1}\right\rangle$. This completes the induction. Now by a direct limit argument we construct a derivation $\vartheta_{j}$ on $Q\langle X, Y\rangle$ such that $\vartheta_{j}\left(A\left\langle Y_{\geqslant 2}\right\rangle\right) \subseteq A\left\langle Y_{\geqslant 2}\right\rangle$.

Finally, since $Q\langle X, Y\rangle=A\left\langle Y_{\geqslant 2}\right\rangle\langle Z\rangle$, for each $u_{k} \in U$, the degree of the element $\vartheta_{j}\left(\partial\left(u_{k}\right)\right)=\vartheta_{j}\left(z_{k}\right)$ dictates that it lie in $A\left\langle Y_{\geqslant 2}\right\rangle$-which is acyclic. Thus, $\partial\left(v_{k}\right)=$ $\vartheta_{j}\left(\partial\left(u_{k}\right)\right)$ for some element $v_{k} \in A\left\langle Y_{\geqslant 2}\right\rangle$, and, by 1.2 we extend $\vartheta_{j}$ to $Q\langle X, Y, U\rangle$ with $\vartheta_{j}\left(u_{k}\right)=(-1)^{\left|\vartheta_{j}\right|} v_{k}$. The $\vartheta_{j}$ thus defined has all the required properties.

Example. Consider the ideal $I=\left(a b, c^{2}, c d, a^{2} c+d^{3}, b^{2} d+a d^{2}\right)$ in the polynomial ring $Q=k[a, b, c, d]$, over a field $k$. The element $a b \in I$ generates a free summand of $I / I^{2}$. Indeed, according to MACAULAY, the beginning of the minimal resolution of $I$ is $Q^{8} \stackrel{\phi}{\longrightarrow} Q^{5} \stackrel{\psi}{\longrightarrow} I \rightarrow 0$ where

$$
\begin{gathered}
\psi=\left(\begin{array}{llllllll}
a b & c^{2} & c d & a^{2} c+d^{3} & b^{2} d+a d^{2}
\end{array}\right), \\
\phi=\left(\begin{array}{cccccccc}
0 & 0 & 0 & -c d & -c^{2} & a^{2} c+d^{3} & a b c & b^{2} d+a d^{2} \\
-d & 0 & a^{2} & 0 & a b & 0 & 0 & 0 \\
c & b^{2}+a d & d^{2} & a b & 0 & 0 & a^{3} & 0 \\
0 & 0 & -c & 0 & 0 & -a b & -b^{2}-a d & 0 \\
0 & -c & 0 & 0 & 0 & 0 & d^{2} & -a b
\end{array}\right) .
\end{gathered}
$$

The entries in the first row in the relations matrix $\phi$ of the ideal $I$ are contained in $I$, so that, by 1.32 , the minimal generator $a b$ generates a free summand in the $Q / I$ module $I / I^{2}$, and thus a free summand in the $R=(Q / I)_{\mathfrak{n}}$-module $\left(I / I^{2}\right)_{\mathfrak{n}}$, where $\mathfrak{n}=(a, b, c, d)$. Now, since $a b \in \mathfrak{n}^{2}$, the Main Theorem yields that $\operatorname{rank}_{k} \zeta^{2}(R) \geq 1$. A propos the question of Avramov, it is of interest to know if the $\mathfrak{n}$-adic completion $\widehat{R}$ has an embedded deformation.

The fibres of flat homomorphisms are a nice source of rings with central elements:

Example. Suppose that $\phi:(R, \mathfrak{m}, k) \rightarrow(S, \mathfrak{n}, l)$ is a flat local homomorphism. Flatness implies that the $S / \mathfrak{m} S$-module $\mathfrak{m} S /(\mathfrak{m} S)^{2}$ is free of rank equal to edim $R$, the embedding dimension of $R$. Therefore, the Main Theorem yields that

$$
\begin{aligned}
\operatorname{rank}_{l} \zeta^{2}(S / \mathfrak{m} S) & \geq \operatorname{edim} R-\operatorname{rank}_{l}\left(\frac{\mathfrak{m} S}{\mathfrak{m} S \cap \mathfrak{n}^{2}}\right) \\
& =\operatorname{edim} R-(\operatorname{edim} S-\operatorname{edim}(S / \mathfrak{m} S)) \\
& =\operatorname{edim} R+\operatorname{edim}(S / \mathfrak{m} S)-\operatorname{edim} S .
\end{aligned}
$$


The same lower bound may be obtained as follows: According to [2, 1.1] (rather, its dual), there is an exact sequence of $l$-vector spaces

$$
0 \rightarrow \pi^{1}(S / \mathfrak{m} S) \rightarrow \pi^{1}(S) \rightarrow \pi^{1}(R) \otimes_{k} l \stackrel{\delta}{\rightarrow} \pi^{2}(S / \mathfrak{m} S) \rightarrow \pi^{2}(S) \rightarrow \pi^{2}(R) \otimes_{k} l \rightarrow 0
$$

where for any local ring $(Q, \mathfrak{q})$, one has $\operatorname{rank}_{Q / \mathfrak{q} Q} \pi^{1}(Q)=\operatorname{edim}(Q)$. The desired inequality follows from the fact that the image of $\delta$ lies in $\zeta^{2}(S / \mathfrak{m} S)$; cf. [8, 1]. This is no coincidence: The central elements detected by the Main Theorem as well as those in $\operatorname{Im}(\delta)$ arise from special cycles.

\section{ON FREE SUMMANDS IN THE CONORMAL MODULE}

The technique utilized in the proof of the Main Theorem leads to another point of view on certain results regarding the conormal module. This is based on the following observation. For a complex $B$ we denote $\sup (B)$ the supremum of the set $\left\{i \mid \mathrm{H}_{i}(B) \neq 0\right\}$; in particular, $\mathrm{H}(B)=0$ if and only if $\sup (B)=-\infty$.

2.1. Proposition. Let $I$ be a proper ideal in a ring $Q$, and let $Q\langle X\rangle$ be a $D G$

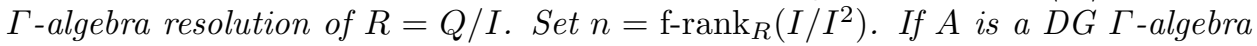
over $Q$ such that $I A_{0} \subseteq \partial\left(A_{1}\right)$, then

$$
A\langle X\rangle=A \otimes_{Q} Q\langle X\rangle \cong W\left\langle z_{1}, \ldots, z_{n} \mid \partial\left(z_{i}\right)=0\right\rangle,
$$

with $W$ a DG $\Gamma$-subalgebra of $A\langle X\rangle$. Therefore, if $\mathrm{H}(A) \neq 0$, then $\sup (A\langle X\rangle) \geq n$.

Proof. Let $x_{1}, \ldots, x_{n}$, respectively $\theta_{1}, \cdots, \theta_{n}$, be the elements in $X_{1}$, respectively the $Q$-linear derivations of $Q\langle X\rangle$, supplied by Proposition 1.4. The derivations $\theta_{i}$ extend to $A$-linear derivations $\widetilde{\theta}_{i}: A\langle X\rangle \rightarrow A\langle X\rangle$, for each $i$.

Since $\partial\left(x_{i}\right) \in I A_{0}$ and $I A_{0} \subseteq \partial\left(A_{1}\right)$, there are elements $a_{1}, \ldots, a_{n}$ in $A_{1}$ such that $\partial\left(a_{i}\right)=\partial\left(x_{i}\right)$ for $1 \leq i \leq n$. Therefore, for each $i$, the elements $z_{i}=x_{i}-a_{i}$ in $A\langle X\rangle$ are cycles of degree 1 . Observe that $\widetilde{\theta}_{i}\left(z_{j}\right)=\delta_{i j}$, that is to say, $z_{1}, \ldots, z_{n}$ are special cycles in $A\langle X\rangle$. Now we invoke the result of André, recalled in 1.6, to obtain the desired isomorphism. It remains to note that if $\mathrm{H}(A) \neq 0$, then $\mathrm{H}_{0}(A) \neq 0$, so that $\mathrm{H}_{0}(A\langle X\rangle)=\mathrm{H}_{0}(A) \otimes_{Q} R=\mathrm{H}_{0}(A) \neq 0$, since $I A_{0} \subseteq \partial\left(A_{1}\right)$. In particular, $\mathrm{H}(W) \neq 0$, and so $\sup (A\langle X\rangle)=\sup (W)+n \geq n$.

Remark. In this note, we shall have occasion to use only the bound $\sup (A\langle X\rangle) \geq n$, and this can be established without resorting to André's result. Indeed, the cycle

$$
z=z_{1} \cdot z_{2} \cdots z_{n} \quad \in \quad A\langle X\rangle_{n}
$$

is not a boundary. This assertion may be justified as follows: $\operatorname{Set} \theta=\widetilde{\theta}_{n} \circ \cdots \circ \widetilde{\theta}_{1}$, and note that $\theta(z)=1$. Now, if $z=\partial(b)$ for some element $b \in A\langle X\rangle_{n+1}$, then

$$
1=\theta(z)=\theta(\partial b)=(-1)^{n} \partial(\theta(b)),
$$

so that $\mathrm{H}_{0}(A\langle X\rangle)=0$, and this implies that $\mathrm{H}(A\langle X\rangle)=0$. This is a contradiction.

The following result has been established, using different techniques, by Vasconcelos [11] and by Ferrand [6]. For a finitely generated $Q$-module $M$, we write $\mu_{Q}(M)$ for the minimal number of generators of $M, \operatorname{grade}_{Q}(I, M)$ for the maximal length of a $M$-regular sequence in an ideal $I \subset Q$, and $\operatorname{pd}_{Q}(M)$ for the projective dimension of $M$ over $Q$. 
2.2. Corollary. Let $I$ be an ideal in a local ring $(Q, \mathfrak{n}, k)$, and set $R=Q / I$. If $\operatorname{pd}_{Q}(R)$ is finite, then

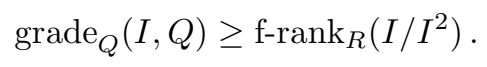

In particular, if $I / I^{2}$ is a free $R$-module, then $I$ is generated by a regular sequence.

Proof. Set $n=\mathrm{f}_{-\operatorname{rank}_{R}}\left(I / I^{2}\right)$, and let $Q\langle X\rangle$ be a DG $\Gamma$-algebra resolution of $R$. Proposition 2.1] gives

$$
\operatorname{pd}_{Q} R=\sup \operatorname{Tor}^{Q}(k, R)=\sup \left(k \otimes_{Q} Q\langle X\rangle\right) \geq n .
$$

In particular, the Auslander-Buchsbaum formula: $\operatorname{depth} Q=\operatorname{pd}_{Q} R+\operatorname{depth} R$, allows us to conclude that $\operatorname{depth} Q \geq n$.

Now, for each prime ideal $\mathfrak{p} \supseteq I$, the $R_{\mathfrak{p}}$-module $I_{\mathfrak{p}} /\left(I_{\mathfrak{p}}\right)^{2}$ contains a free summand of rank $n$. Since $\operatorname{pd}_{Q_{\mathfrak{p}}} R_{\mathfrak{p}}$ is finite as well, we deduce that depth $Q_{\mathfrak{p}} \geq n$, and hence

$$
\operatorname{grade}_{Q}(I, Q)=\inf \left\{\operatorname{depth} Q_{\mathfrak{p}} \mid \mathfrak{p} \supseteq I\right\} \geq n .
$$

Finally, if $I / I^{2}$ is free, then $\mathrm{f}_{-\operatorname{rank}_{R}}\left(I / I^{2}\right)=\mu(I)$, so that $\operatorname{grade}_{Q}(I, Q)=\mu_{Q}(I)$, and hence $I$ is generated by a regular sequence.

The next result also has predecessors: Vasconcelos [11] and Lech [9] have proved, using different techniques, that if $I / I^{2}$ is a free $R$-module, then $\mu_{Q}(\mathfrak{n}) \geq \mu_{Q}(I)$.

2.3. Corollary. Let $I$ be an ideal in a local ring $(Q, \mathfrak{n}, k)$, and set $R=Q / I$. For each proper ideal $J \supseteq I$, one has

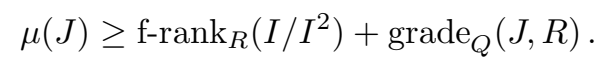

Proof. Let $Q\langle X\rangle$ be a DG $\Gamma$-algebra resolution of $R$, and let $K$ be the Koszul complex on a minimal set of generators for $J$. Since $K$ is a bounded complex of free $Q$-modules, the canonical map $K \otimes_{Q} Q\langle X\rangle \rightarrow K \otimes_{Q} R$ is a quasi-isomorphism. As $I \subseteq J=\partial\left(K_{1}\right)$, Proposition 2.1 yields

$$
\sup \left(K \otimes_{Q} R\right)=\sup \left(K \otimes_{Q} Q\langle X\rangle\right) \geq \mathrm{f}-\operatorname{rank}_{R}\left(I / I^{2}\right) .
$$

It remains to note that, since the Koszul complex is depth sensitive, $\mu_{Q}(J)=$ $\operatorname{grade}_{Q}(J, R)+\sup \left(K \otimes_{Q} R\right)$.

\section{ACKNOWLEDGMENT}

It is a pleasure to thank Luchezar Avramov for kind encouragement, and for useful conversations regarding the material presented here.

\section{REFERENCES}

[1] M. André, Le caractère additif des déviations des anneaux locaux, Comment. Math. Helv. 57 (1982) 648-675. MR 85a:14006

[2] L. Avramov, Homology of local flat extensions and complete intersection defects, Math. Ann. 228 (1977), 27-37. MR 58:5638

[3] L. Avramov, Infinite free resolutions, Six lectures on commutative algebra, Belaterra 1996 (J. Elias, J. Giral, M.-R. Miró-Roig, S. Zarzuela, eds.), Progr. Math. 166, Birkhäuser, Basel, 1998; 1-118. CMP 99:02

[4] L. Avramov, Homological asymptotics of modules over local rings, Commutative algebra; Berkeley, 1987 (M. Hochster, C. Huneke, J. Sally, eds.), MSRI Publ. 15, Springer, New York 1989; 33-62. MR 90i:13014

[5] N. Dupont, Un diviseur de zéro induisant un élément d'homotopie central, Bull. Soc. Math. France 125 (1997), 337-344. MR 99a:55008 
[6] D. Ferrand, Suite régulière et intersection complète, C. R. Acad. Sci. Paris Sèr. A 264 (1967), 427-428. MR 36:2626

[7] T. Gulliksen, G. Levin, Homology of local rings, Queen's Papers Pure Appl. Math. 20, Queen's Univ., Kingston, ON, 1969 MR 41:6837

[8] C. Jacobsson, On local flat homomorphisms and the Yoneda Ext-algebra of the fibre, Astérisque 113-114 (1984), 227-233. MR 86c:13011

[9] C. Lech, A property of independent elements, J. Pure Appl. Algebra 38 (1985), 285-286. MR 87e:13016

[10] C. Löfwall, Central elements and deformations of local rings, J. Pure App. Algebra 91 (1994), 183-192. MR 95g:13018

[11] W. V. Vasconcelos, Ideals generated by R-sequences, J. Algebra 6 (1967), 309-316. MR 35:4209

Department of Mathematics, University of Missouri, Columbia, Missouri 65211

E-mail address: iyengar@math.missouri.edu

Current address: Department of Pure Mathematics, University of Sheffield, Hicks Building, Sheffield S3 7RH, United Kingdom

E-mail address: s.iyengar@shef.ac.uk 